\title{
Cellular contaminants of adeno-associated virus vector stocks can enhance transduction
}

\author{
L Tenenbaum ${ }^{1,2}$, M Hamdane ${ }^{1}$, M Pouzet ${ }^{1,2}$, B Avalosse ${ }^{3}$, A Stathopoulos ${ }^{1,2}$, F Jurysta ${ }^{2}$, \\ C Rosenbaum ${ }^{4}, \mathrm{CO}$ Hanemann $^{4}, \mathrm{M}$ Levivier $^{2}$ and $\mathrm{T}$ Velu $^{1}$ \\ ${ }^{1}$ IRIBHN, Campus Erasme, Université Libre de Bruxelles; ${ }^{2}$ Department of Neurosurgery, Hôpital Erasme, Université Libre de \\ Bruxelles; ${ }^{3}$ Laboratory of Molecular Biology, Institut Bordet, Université Libre de Bruxelles and ${ }^{4}$ Department of Neurology, Heinrich \\ Heine University, Düsseldorf, Germany
}

Transduction efficiency of different types of recombinant (r)AAV-2 based vectors preparations markedly differed, with apparently no correlation with the replicative titers. Using HeLa cells as target for transduction, $10^{5}$ and 30 infectious units were necessary to observe one transductant using respectively cesium-chloride-purified rAAV and crude lysates of producer cells obtained by sonication. The purified vectors were however able to transduce HEK-193 cells efficiently, but transgene expression was detected with some delay compared with crude lysates. The unexpected high transduction efficiency of sonicated crude lysates was due to virally mediated gene transfer, since similar sonicated crude lysates, but with no AAV rep and cap genes, did not lead to detection of transgene products after incubation with HeLa cells. Furthermore, sonicated cellular extracts of 293 or 293/T cells given in trans stimulate transduction of HeLa cells by purified rAAV. In contrast, neither extracts from the adenovirus E1-transformed 911 cell line, nor from other cell lines not harboring any adenovirus gene, had enhancing effect on rAAV-mediated transduction. These data suggest that 293 sonicated extracts contain factors which stimulate $r A A V$-mediated transduction of cells that are normally poorly transduced and offer a system to identify such factors and to characterize further the steps limiting the transfer of gene by $A A V$ vectors.

Keywords: adeno-associated virus; gfp; contaminants; titration; brain

\section{Introduction}

Wild-type AAV type 2 life cycle is dependent on cellular as well as helper virus factors at several stages including intracellular compartimentalization, ${ }^{1}$ DNA replication, ${ }^{2-4}$ gene expression ${ }^{5}$ and encapsidation. ${ }^{6}$ The fact that adenovirus, vaccinia virus or herpes simplex virus infection, ${ }^{7}$ UV light, carcinogens $s^{3,8}$ and cell synchronization ${ }^{2}$ stimu- $^{-}$ late AAV replication to different extents, suggests that key cellular factor(s) induced by these treatments are necessary to trigger AAV replication. In addition, the adenovirus DNA-binding protein has recently been shown to provide a direct helper effect for in vitro replication of double-stranded AAV genomes. ${ }^{4}$

Recombinant AAV type 2-mediated gene transfer requires virus attachment and internalization, migration to the nucleus, decapsidation, conversion to doublestranded DNA and gene expression. At least some of these steps are also dependent on cellular factors and can be triggered by adenovirus, ${ }^{4,9,10}$ hydroxyurea, ${ }^{11} \mathrm{X}$ rays, ${ }^{12}$ serum-deprivation $^{13}$ or agents that interfere with DNA metabolism. ${ }^{13}$ Conversion of viral single-stranded DNA to transcriptionally active double-stranded DNA is thought to be one of the limiting steps for transduction. ${ }^{9,10}$ Second-strand synthesis has been correlated with

Correspondence: L Tenenbaum, IRIBHN, Bât C, Campus Erasme, Université Libre de Bruxelles 808, Route de Lennik, B-1070 Brussels, Belgium Received 20 October 1998; accepted 15 January 1999 the dephosphorylated state of a cellular factor binding to the $20 \mathrm{bp} D$ sequence that juxtaposes the $125 \mathrm{bp}$ terminal palindromes in each of the $145 \mathrm{bp}$ terminal repeats. ${ }^{14}$ The level of dephosphorylation of this ' $D$ binding protein' (DBP) varies from one cell line to the other ${ }^{14,15}$ and from one tissue to the other ${ }^{16}$ and correlates with the transduction efficiency in the given cell line or tissue.

Conflicting results exist in the literature regarding the efficiency of rAAV-mediated transduction of various cell types, in particular non-dividing cells (as discussed in Alexander et $\mathrm{l}^{17}$ ). Some of these discrepancies can at least partly be explained by 'pseudotransduction', a phenomenon consisting of artefactual transfer of gene products through non-purified viral lysates rather than true gene transfer and expression of virally encoded gene products. ${ }^{17}$

In this report, we showed that although purified viral particles poorly transduce HeLa cells, some crude, nonpurified viral preparations can transfer and express the transgene in these cells through a virally mediated mechanism, in the absence of pseudotransduction. We further showed that sonicated lysates made from untransfected 293 cells can stimulate transduction when incubated with HeLa cells together with a CsCl-purified rAAV viral preparation.

\section{Results}

Sonicated crude lysates of rAAV-EGFP transduce HeLa cells $10^{4}$-fold better than purified virus

An AAV plasmid vector harboring the 'enhanced humanized gfp' (EGFP) gene as reporter transgene under 


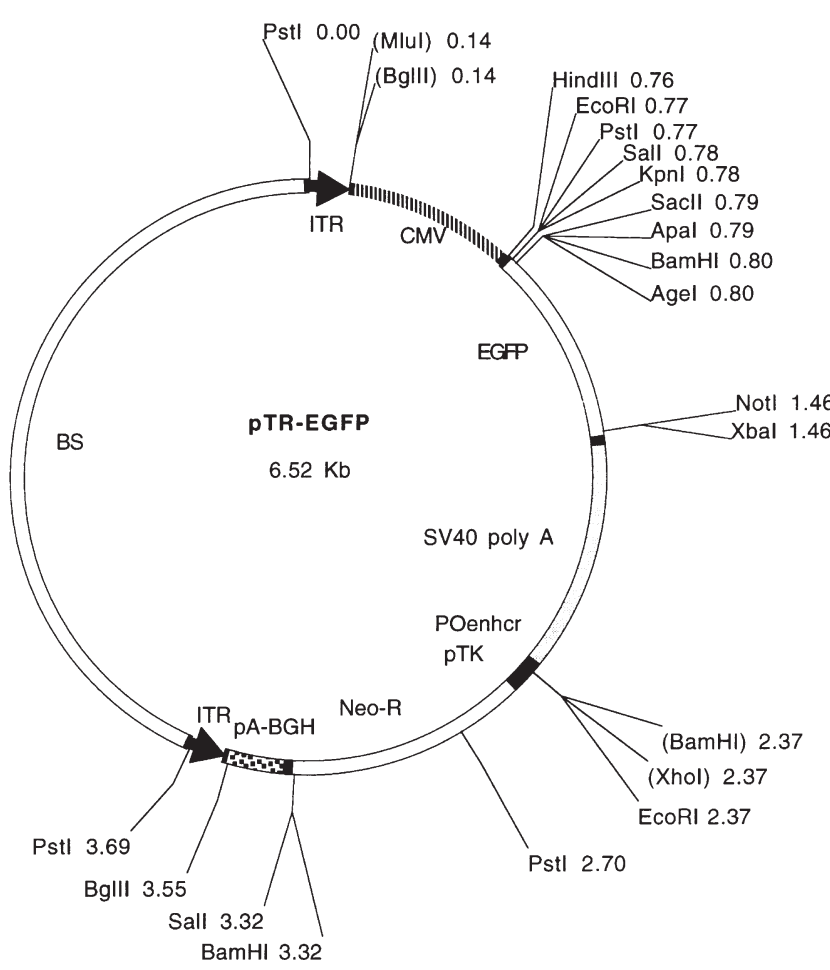

Figure 1 Map of pTR-EGFP. BS, pBlue-Script (Stratagene, La Jolla, CA, USA); ITR, AAV inverted terminal repeats (map position 1-145); CMV cytomegalovirus promoter (from $p$ GFPS65T, Clontech); EGFP, enhanced GFP (from $p E G F P-1$, Clontech); SV40 polyA, SV40 polyadenylation signal and SV40 intron (from pGFPS65T, Clontech); POenher, polyoma enhancer (from $p T R-U F$ ) ${ }^{37}$ pTK, herpes simplex virus thymidine kinase (HSVtk) promoter (from $p T R-U F) ;{ }^{37}$ Neo-R, neomycin-resistance gene (from $p T R-U F) ;{ }^{37} p A-B G H$, polyadenylation signal from bovine growth hormone gene (from $p T R$-UF). ${ }^{37}$

the control of the cytomegalovirus (CMV) promoter (see Figure 1) and HeLa cells as targeted cells, were used in order to determine the transduction efficiency of rAAV viral preparations.

The rAAV-EGFP viral preparations were titrated by in situ focus hybridization assay on HeLa cells. The titers of crude lysates varied in the range of $3 \times 10^{6}$ to $10^{7}$ infectious units (IU)/ml (see Figure $2 \mathrm{~b}$ as an example). The titers of purified stocks varied between $10^{8}$ and $5 \times 10^{8}$ $\mathrm{IU} / \mathrm{ml}$. The contamination by wild-type AAV was evaluated by omitting AAV coinfection and hybridizing with

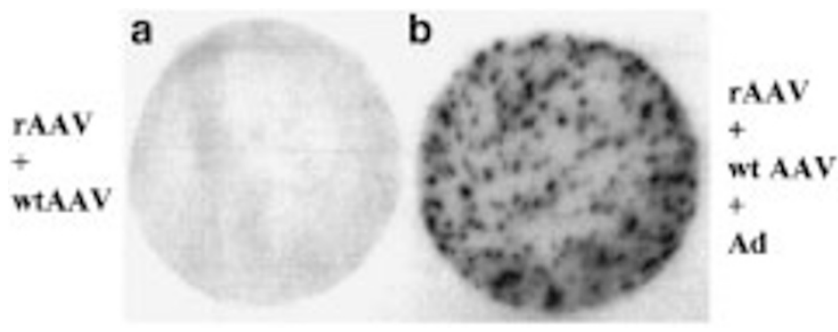

Figure 2 Titration of $r A A V$-EGFP viral stocks. (a) HeLa cells were coinfected with wild-type AAV (200 IU per cell) and $10 \mu \mathrm{l}$ of undiluted sonicated rAAV-EGFP crude lysate. (b) HeLa cells were co-infected with adenovirus (100 p.f.u. per cell), wild-type AAV (200 IU per cell) and $2 \mu l$ of a 1:100 dilution of the sonicated crude lysate. The replicative titer of this stock was $10^{7} \mathrm{IU} / \mathrm{ml}$. an AAV probe lacking the ITRs and was found to be less than $10^{-3} \mathrm{IU} / \mathrm{rAAV}-\mathrm{EGFP}$ IU (data not shown).

For the evaluation of the transduction efficiency, $2 \times$ $10^{4} \mathrm{HeLa}$ cells were infected with $10^{5} \mathrm{IU}$ of crude lysate or purified rAAV/EGFP virus. Green fluorescent cells were counted under UV microscopy. Crude lysates could efficiently transduce HeLa cells (Figure 3 and Table 1). In contrast, purified virus had a very low transduction efficiency (less than five fluorescent cells per well; Table 1).

\section{Sonicated crude lysates of rAAV-EGFP transduce 293 cells more rapidly than purified virus}

To determine if the difference between the transduction efficiency of purified and crude lysates of rAAV-EGFP observed in HeLa cells also appears in other cell lines, we infected HEK-293 with the two types of viral preparations using the same multiplicity of infection (5 IU per cell).

Similarly to HeLa cells, 2 days after infection, crude lysates of rAAV-EGFP transduce HEK-293 cells with a high efficiency whereas very few $\mathrm{gfp}^{+}$cells were observed when using purified virus (Table 1). However, 5 days after infection with purified virus, numerous $\mathrm{gfp}^{+}$ HEK-293 cells appeared. On the contrary, only very few (less than 10) HeLa cells were $\mathrm{gfp}^{+}$, even after 5 days (data not shown).

\section{Sonicated crude lysates of rAAV-EGFP do not contain functional adenovirus}

Adenovirus is known to stimulate rAAV-mediated transduction. ${ }^{9,10}$ Adenoviral contamination could thus account for the observed high transduction efficiency of the sonicated crude lysates. Therefore, we tested if the heating treatment efficiently inactivated the helper adenovirus still present in the crude preparations. A large amount of a sonicated crude lysate ( $10^{5}$ IU AAV-EGFP virus) was used to infect HeLa cells in the presence of wild-type AAV but in the absence of exogeneously added adenovirus. The cell monolayer was transferred to a nitrocellulose filter as described for the titration (see Materials and methods). No autoradiographic spot was observed after hybridization with a DNA probe for the Egfp gene, showing that the sonicated crude lysates do not contain functional adenovirus after heat inactivation (Figure 2a).

To confirm further the absence of residual infectious adenovirus in the rAAV-EGFP crude and purified viral stocks, infected HeLa or HEK-293 cells were further cultured and compared with uninfected cells. There was no apparent cytopathic effect in the infected cultures until 2 weeks after infection (data not shown).

\section{In vivo transduction efficiency in the rat central nervous} system

In order to test the hypothesis that rAAV-EGFP particles had been damaged or had lost a co-factor necessary for transduction during the purification process, we injected a purified viral preparation in the rat brain parenchyma, a tissue for which numerous reports of efficient reporter gene expression have been published using AAV vectors. ${ }^{12,18-24}$ One month after injection of $2 \times 10^{5} \mathrm{IU}$ of rAAV-EGFP, numerous cells with neuron morphology were transduced in the globus pallidus (Figure 4). No immunostained cell was observed in the animals injected with PBS, showing the specificity of the labeling (data not 


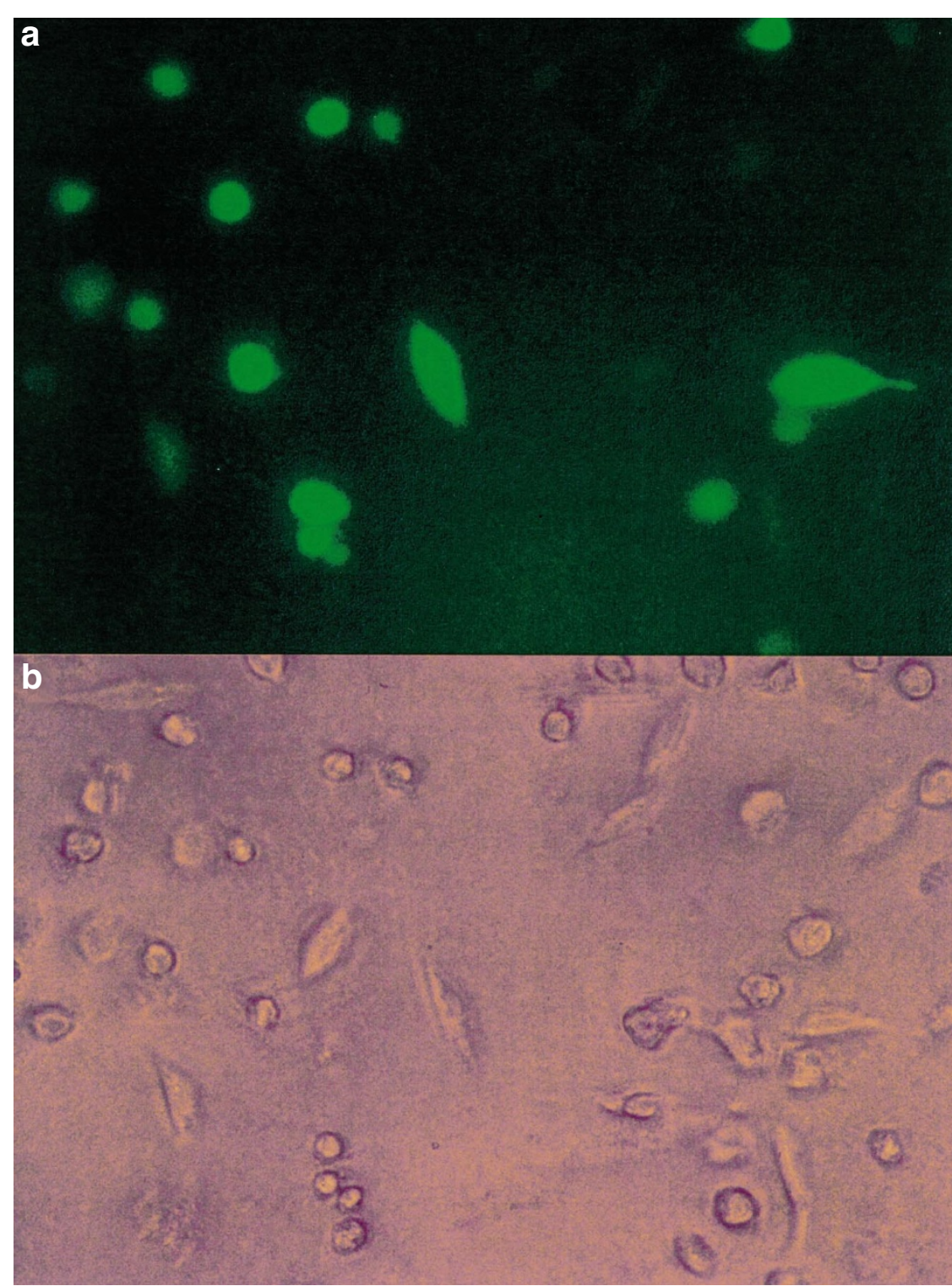

Figure 3 Transduction efficiency of $r A A V$-EGFP sonicated crude lysate. Twenty-thousand HeLa cells were infected with $10^{5}$ IU of $r A A V$-EGFP sonicated crude lysate $(20 \mu l)$. Forty-eight hours after infection, the cells were photographed under UV (a) or visible light (b).

shown). These data indicate that our purified rAAVEGFP is functional.

\section{EGFP-encoded protein or nucleic acid contaminants of crude viral preparations do not account for high transduction efficiency}

Alexander et $\mathrm{l}^{17}$ recently showed that transgene-encoded protein contaminants present in non-purified AAV vector preparations can be taken up by cells exposed to the vectors and mimic true vector-mediated transduction, a phenomenon designated as 'pseudotransduction'. This phenomenon has been observed with the LacZ and the alkaline phosphatase reporter genes. ${ }^{17}$ In our experiments, difference between the transduction efficiency of sonicated crude lysates and purified rAAV-EGFP virus could thus be due to pseudotransduction. To test this hypothesis, 'pseudostocks' containing EGFP-encoded contaminants were prepared following a protocol identical to the preparation of sonicated crude lysate except that the pIM45 plasmid carrying the wild-type AAV coding region, was omitted in the transfection of HEK293 cells (see Materials and methods). HeLa cells were infected with pTR-EGFP pseudostock, rAAV-EGFP sonicated crude lysate or with purified rAAV-EGFP. The number of green fluorescent cells was evaluated by fluorescence activated cell sorter (FACS) analysis.

No green fluorescent cell was observed when cells were incubated with these pseudostocks (Figure 5 column 3), while, as expected, the transduction efficiency was high for the rAAV-EGFP crude lysates (Figure 5 column 2) and low for the purified viral vectors (Figure 5 column 1).

Thus crude lysates prepared in the absence of Rep and Cap proteins do not transfer EGFP gene products to HeLa cells.

However, in cells transfected with the pTR-EGFP plasmid alone, the level of gfp protein could be lower than in cells transfected with both pTR-EGFP and pIM45, since 
Table 1 Post-infection time dependence of transducing titer in 293 cells and HeLa cells

\begin{tabular}{lccllll}
\hline \multirow{2}{*}{$\%$ gfp cells } & \multicolumn{2}{c}{ HeLa cells } & & \multicolumn{2}{c}{293 cells } \\
\cline { 2 - 3 } \cline { 6 - 7 } \cline { 5 - 6 } & 2 days & 5 days & & 2 days & 5 days \\
\hline $\begin{array}{l}\text { Crude lysate } \\
\begin{array}{l}\text { (sonication) } \\
\text { Purified }(\mathrm{CsCl})\end{array}\end{array}$ & 31.6 & $\mathrm{ND}$ & & 51.4 & $\mathrm{ND}$ \\
\hline
\end{tabular}

Six crude lysates and three purified preparations were analyzed. The titers (evaluated by in situ focus hybridization assay) were $(5.5 \pm 3.3) \times 10^{6}$ for the crude lysates and $(2.1 \pm 1.7) \times 10^{8}$ for purified stocks. One hundred thousand infectious units were used to infect $2 \times 10^{4} \mathrm{HeLa}$ cells in the absence of helper virus. The transduction efficiency was evaluated by counting the fluorescent cells under UV microscopy and the total number of cells under visible light microscopy, in given representative areas. Data from one representative experiment are shown. $\mathrm{ND}$, not determined.

in the latter case, the rAAV-EGFP genome replicates. To test if a higher number of gfp-encoding genomes in the crude lysates could give rise to pseudotransduction, 293 cells were infected with a recombinant adenovirus encoding gfp (rAd-EGFP) at a multiplicity of 3 p.f.u. per cell. When extracts prepared by sonication and heat-inactivated at $56^{\circ} \mathrm{C}$ were incubated with HeLa cells, no gfppositive cell was observed (Figure 5 column 4).

To characterize further the EGFP gene transfer in HeLa cells by sonicated crude lysates, the number of viral stocks and the time of incubation were varied. When decreasing amounts of sonicated crude lysate or of purified rAAV-EGFP viral stocks were used to infect HeLa cells, the proportion of transduced cells decreased proportionally, which is consistent with a virus-mediated gene transfer (see Figure $6 a$ and $b$ ). However, the absolute level of transduction was more than one order of magnitude lower with purified virus than with crude lysates.

The transduction efficiency of crude lysates increased with time after infection. Indeed, no gfp ${ }^{+} \mathrm{HeLa}$ cell was observed at $24 \mathrm{~h}$ after infection, whereas $35.5 \pm 6.7 \%(n=$ $3)$ and $55.5 \pm 8.8 \%(n=4)$ of gfp ${ }^{+}$cells at $48 \mathrm{~h}$ and $72 \mathrm{~h}$, respectively, were observed at a multiplicity of infection of 3. Thus, as expected for virally mediated gene transfer, the number of transduced cells increased with time after infection.

\section{Enhancement of transduction by sonicated cellular extracts}

The higher transduction efficiency of sonicated crude lysates as compared to purified virus could be due to the transfer of a HEK-293 cellular factor stimulating the susceptibility of HeLa cells to rAAV-mediated transduction. To test this hypothesis, HeLa cells were co-incubated with purified rAAV-EGFP and sonicated, heat-treated extracts of untransfected, uninfected HEK-293 cells prepared according to the same protocol as for the crude viral lysates (see Materials and methods). Two days after infection, the percentage of $\mathrm{gfp}^{+}$cells was determined by FACS analysis (Figure 7). In the absence of cellular extract, the transduction efficiency of purified virus was low: $0.6 \%$ (Figure 7, lane 1). In the presence of HEK-293
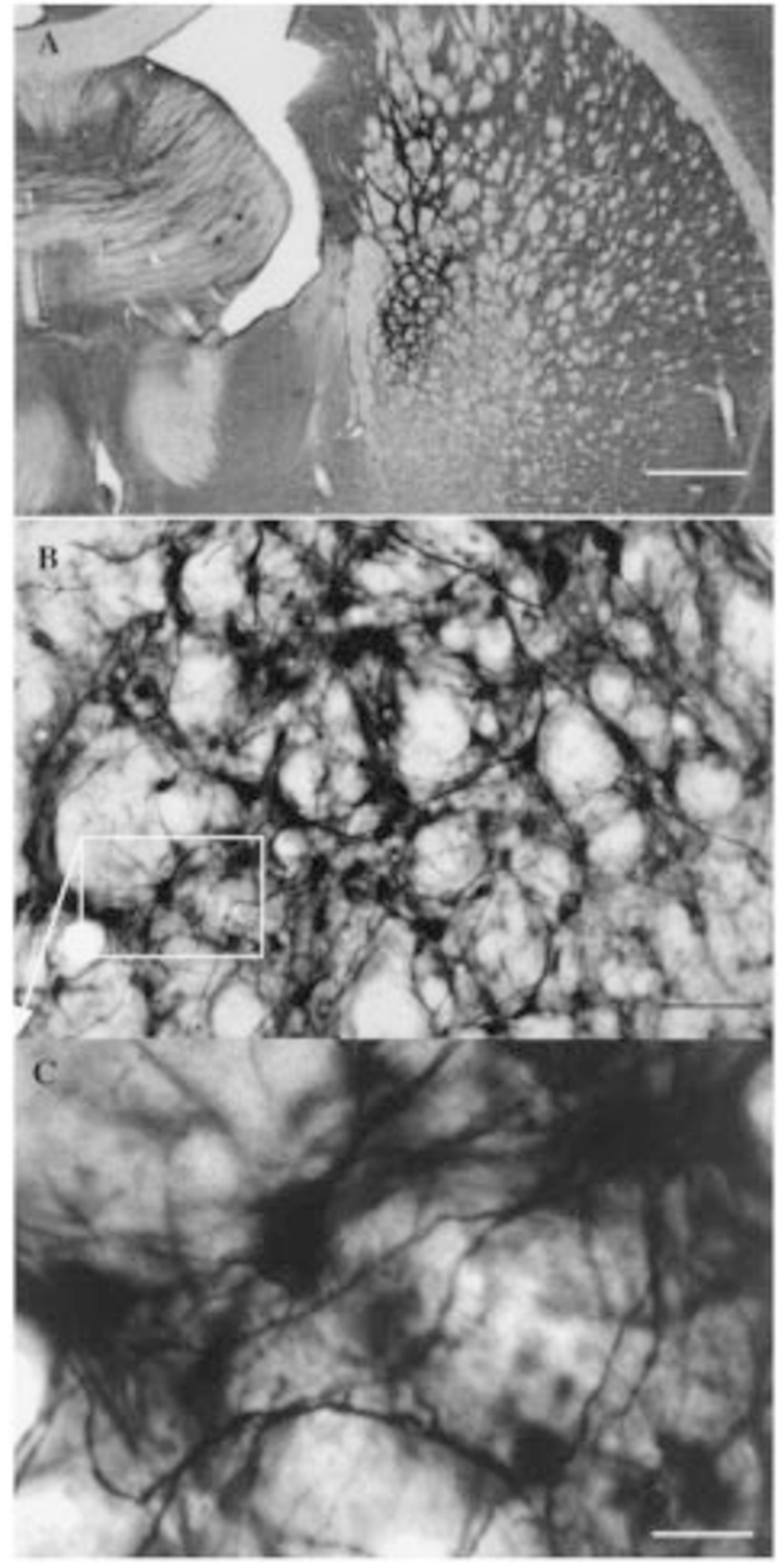

Figure 4 In vivo transduction efficiency. Two $10^{5}$ IU of $r A A V$-EGFP purified virus in $2 \mu l$ were injected into the rat globus pallidus. One month after injection, vibratome sections were analyzed by immunohistochemistry using an anti-gfp antibody. The bar corresponds to $800(A)$, $100(B)$ and $20 \mu \mathrm{m}(\mathrm{C})$, respectively.

cellular extract, the transduction efficiency of purified rAAV-EGFP markedly increased: $9.6 \%$ of the cells were fluorescent (Figure 7, lane 2). The percentage of gfp ${ }^{+}$cells was however lower when the HEK-293 cellular extract was present in trans together with purified virus (about $10 \%)$, than when using rAAV-EGFP sonicated crude lysates (about 25\%; Figure 7; lane 13). Adenovirus added in trans stimulated transduction to an even greater extent (about 40\% Figure 7; lane 12). Therefore, we evaluated the possible role of inactivated adenovirus in further stimulating transduction. When sonicated heat-treated 


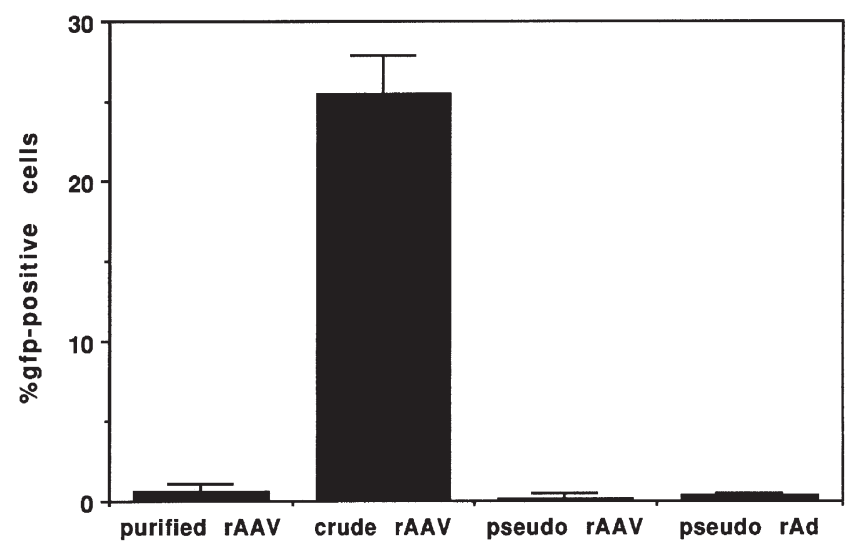

Figure 5 Lack of pseudotransduction by crude lysates of pTR-EGFPtransfected HEK-293 cells. Fifty thousand cells were incubated with 2.0 $\times 10^{5} \mathrm{IU}$ of purified $r A A V$-EGFP (column 1), $2.0 \times 10^{5} \mathrm{IU}(100 \mu \mathrm{l})$ of rAAV-EGFP sonicated crude lysate (column 2), $100 \mu$ l of crude lysate from 293 cells transfected with $p T R-E G F P$ and infected with adenovirus (column 3) or $100 \mu \mathrm{l}$ of crude lysate from 293 cells infected with rAdEGFP at a multiplicity of 3 p.f.u. per cell (column 4). Two days after infection, $10^{4}$ cells were analyzed by FACS. Average values \pm s.e.m. are shown $(n=3)$.

extracts of HEK-293 cells infected with adenovirus (Figure 7, lane 4), were incubated in the presence of purified rAAV-EGFP no increase in the percentage of HeLa fluorescent cells was observed as compared with the trans effect of 'naive' HEK-293 cell extracts (Figure 7, lane 2). To characterize further the requirements for in trans stimulation of purified rAAV-EGFP transduction efficiency, we tested the ability of sonicated extracts from other cell lines to stimulate transduction of HeLa cells by purified rAAV. Heat-treated extracts from HeLa cells (Figure 7, lane 8) and CV1 cells (Figure 7, lane 11) were negative. Extracts from 293/T (SV40 T antigen transformed 293 cell line) could stimulate transduction to the same extent as 293 cells (Figure 7, lane 6). In contrast, extracts from 911 cells (adenovirus E1-transformed human retinoblasts) fail to stimulate transduction (Figure 7 , lane 7). The lack of transduction stimulation by 911 cells further suggests that the presence of adenovirus $\mathrm{E} 1 \mathrm{~A}$ and $\mathrm{E} 1 \mathrm{~B}$ genes in the sonicated cell extract is not sufficient to trigger rAAV-mediated transduction. Adenoviral genes distinct from E1A and E1B and present in 293 cells could play a role in the observed stimulation of transduction. To study the potential role of adenovirus further, HeLa cells were infected by adenovirus 2 at a multiplicity of 100 p.f.u. per cell. Extracts from adenovirus 2-preinfected HeLa cells did not stimulate transduction efficiency by purified rAAV-EGFP (Figure 7, lane 9), suggesting that adenovirus genes were not directly responsible for the observed enhancement.

Rep proteins present in higher concentration in the non-purified virus preparations could favor conversion to transcriptionally active double-stranded DNA. ${ }^{32}$ Because efficient expression of Rep proteins requires adenovirus infection, ${ }^{5}$ we transfected adenovirus-preinfected HeLa cells with pIM45. Rep proteins expression did not potentiate HeLa cell extracts (Figure 7, lane 10). Similarly, extracts from HEK-293 cells transfected with pIM45 (Figure 7, lane 3), or both transfected with pIM45 and infected with adenovirus (Figure 7, lane 5) did not
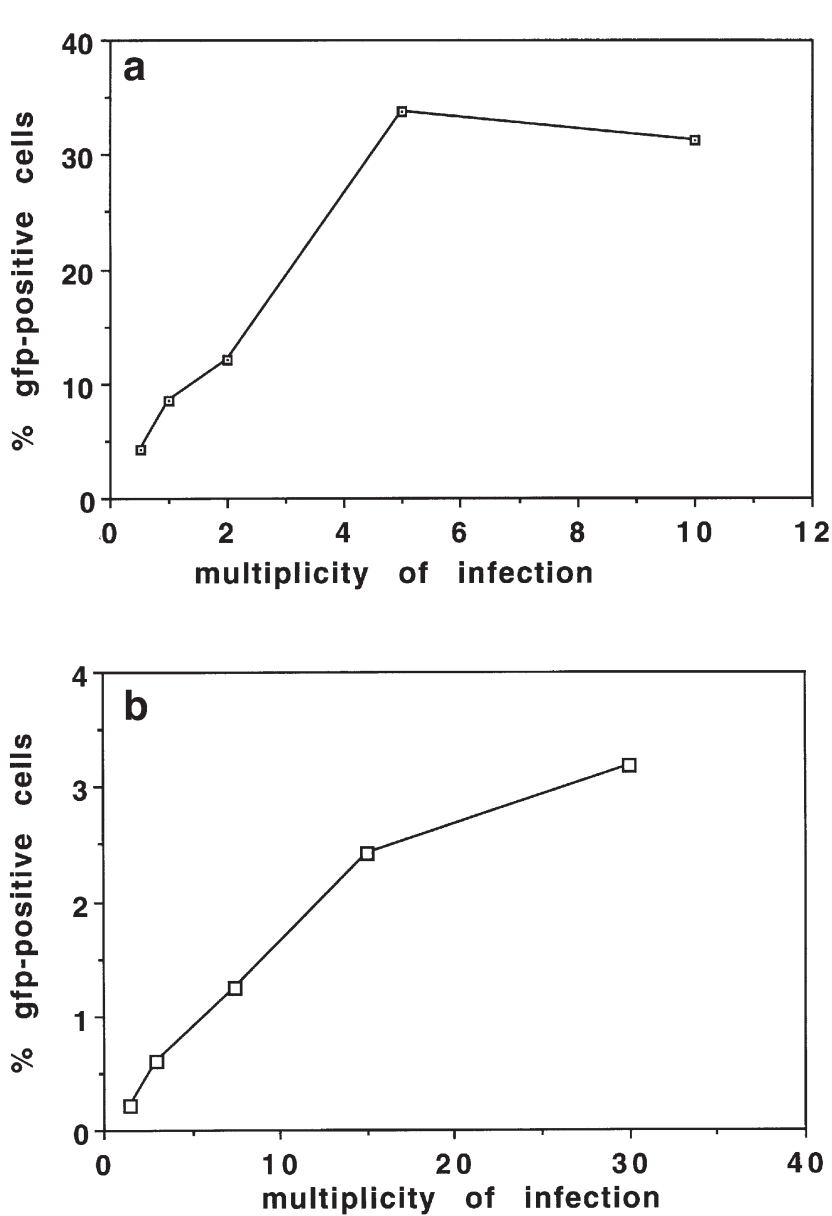

Figure 6 Transduction efficiency of rAAV-EGFP purified versus sonicated crude lysate preparations at different multiplicities of infection. (a) Fifty thousand cells were infected with 5, 10, 20, 50 and $100 \mu \mathrm{l}$ (corresponding to a multiplicity of $0.5,1,2,5$ and $10 \mathrm{IU}$ per cell, respectively) of a sonicated crude lysate of $r A A V$-EGFP with a titer of 5 $\times 10^{6} \mathrm{IU} / \mathrm{ml}$. Sonicated crude lysate of untransfected 293 cells was added up to $100 \mu \mathrm{l}$. Two days after infection, cells are trypsinized and $10^{4}$ cells were analyzed by FACS. (b) Fifty thousand cells were infected with 5, 10, 25, 50 and $100 \mu \mathrm{l}$ (corresponding to a multiplicity of 1.5, 3, 7.5, 15 and $30 \mathrm{IU}$ per cell, respectively) of $\mathrm{CsCl}$-purified, non-microconcentrated $r A A V$-EGFP with a titer of $2 \times 10^{7} \mathrm{IU} / \mathrm{ml}$. Two days after infection, cells were trypsinized and $10^{4}$ cells were analyzed by FACS. Data from one out of three representative experiments are shown.

stimulate transduction to a greater extent than naïve HEK-293 extracts.

\section{Discussion}

With the emergence of recombinant AAV as a potential key vehicle for gene transfer, major efforts have been invested in improving techniques to produce, ${ }^{26-31}$ purify and concentrate ${ }^{27,28}$ viral AAV vectors. However, the interpretation of data on transduction of various cell types and organs has been complicated by the wide variations existing between the purity and concentration of viral stocks used by different authors. Discrepancies between data obtained with rAAV crude lysates and purified virus concerning for example the efficiency of gene transfer in non-dividing fibroblasts have been recently pointed out by Alexander et al. ${ }^{17}$ The authors show that the efficient transgene expression observed by Podsakoff 
$\%$ gfp-positive cells

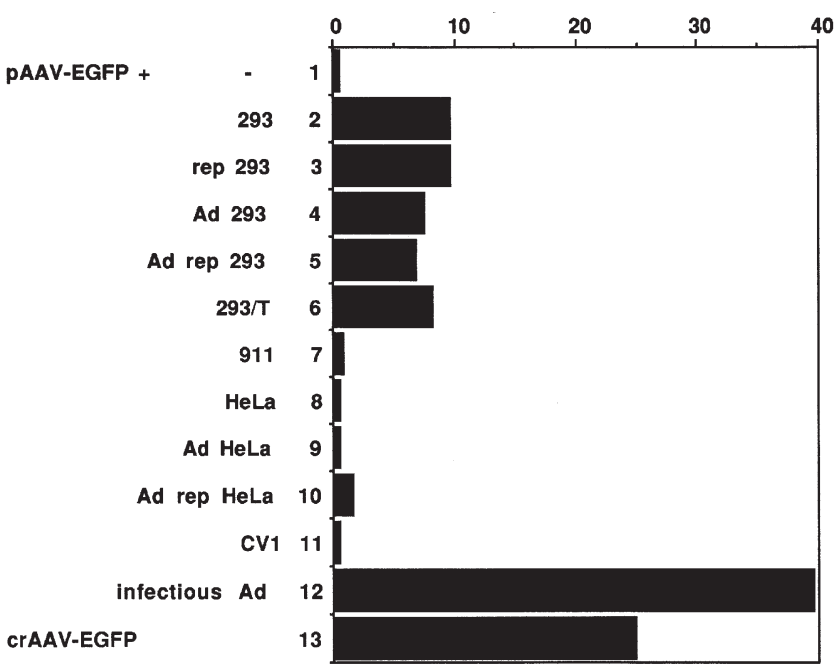

Figure 7 In trans stimulation of $r A A V$-EGFP transduction efficiency by sonicated cellular extracts. Fifty thousand HeLa cells were incubated with $10 \mu l$ of purified $r A A V$-EGFP corresponding to $2 \times 10^{5}$ IU (lanes 1-10) and $100 \mu \mathrm{l}$ of sonicated heat-treated cellular extracts from 293 (lanes 25), 293/T (lane 6), 911 (lane 7), HeLa (lanes 8-10) or CV1 (lane 11) cells. Positive controls: HeLa cells were incubated with $10 \mu$ l of purified $r A A V$ EGFP and 100 p.f.u. per cell of wild-type infectious adenovirus (lane 12) or with $100 \mu \mathrm{l}$ of sonicated heat-treated AAV-EGFP producer cells (crude viral lysate; lane 13). Two days after infection, cells were trypsinized and $10^{4}$ cells were analyzed by FACS. Data from one of three representative experiments are shown.

et $a l^{25}$ and not by Russell et $a l^{11}$ can be explained by an artefact that they called 'pseudotransduction', consisting of the uptake of transgene products expressed by the vector plasmid after transfection into producer cells, rather than by virus-mediated transgene expression. This phenomenon has been observed in two different cell types, $\mathrm{CD} 4^{+}$cells and fibroblasts, using two different reporter genes: $L a c Z$ and $A P$ (alkaline phosphatase). The data reported in this paper, suggest that discrepancies between data obtained using viral preparations with different degrees of purity could also be related to a higher transduction efficiency observed with crude lysates as compared with purified recombinant virus.

The transduction efficiencies of purified viral preparations and of crude lysates prepared from cells producing a rAAV vector expressing the EGFP gene, have been compared. The different viral preparations were titrated using the in situ focus hybridization assay which reveals replication-competent viral particles. ${ }^{3}$ Clark et al ${ }^{32}$ and Salvetti et $a l^{33}$ using purified rAAV virus, reported that, when evaluated in the presence of helper adenovirus, the transduction titer is always lower than the replicative titer. We found that using the same number of replicative particles and in the absence of infectious adenovirus, the transduction efficiency of cesium-chloride purified rAAV-EGFP virus is drastically lower than that of sonicated crude lysates. Indeed, at a multiplicity of 5 infectious units per cell, about $0.5 \%$ of HeLa cells were gfp $^{+}$ using purified virus whereas about $30 \%$ of gfp $^{+}$cells were observed using sonicated crude lysates. The loss of transduction efficiency which accompanied purification was not due to a loss of integrity of viral capsids, since the purified virus was still able to transfer the EGFP gene in HEK-293 cells efficiently, as well as in the rat cerebral parenchyma. However, the time required to observe the expression of the transgene in HEK-293 cells was longer when using purified virus than crude lysates.

Transduction of HeLa cells and 293 cells by purified rAAV in the absence of adenovirus, has been repeatedly reported in the literature. $9,10,30,32,33$ The transduction efficiency of HeLa cells greatly varied according to the different authors. These discrepancies could be due to different input multiplicities and/or to the use of different batches of cells. Consistently with our results, in one of these reports comparing the two cell lines, 293 cells are transduced with a significantly higher efficiency than HeLa cells. ${ }^{32}$

A possible role of pseudotransduction ${ }^{17}$ has been evaluated to explain the high transduction efficiency observed using sonicated crude lysates in vitro. Sonicated crude lysates of adenovirus-infected HEK-293 producer cells transfected with the pTR-EGFP AAV vector plasmid, but not with the helper plasmid harboring AAV rep and cap viral genes necessary for recombinant virus replication and encapsidation, were prepared. When HeLa cells were incubated in the presence of these 'pseudocrude lysates', mimicking the viral adsorption step, no significant increase in the background fluorescence of the cells was observed. This is in contrast to the report by Alexander et $a l^{17}$ showing that crude lysates of rAAV viral vectors expressing the alkaline phosphatase (AP) or the $L a c Z$ reporter gene can transfer contaminants to infected cells, resulting in an artefactual detection of gene transfer. Furthermore, if the high transduction efficiency observed with our sonicated crude lysates originated from the uptake of contaminants, one would expect that, by decreasing the amount of crude preparation used to infect HeLa cells, the fluorescence would be fading in all cells. In contrast, if the observed fluorescence originated from virally mediated EGFP gene expression, the number of fluorescent cells would decrease proportionally to the input virus, with positive cells keeping the same intensity. In our experimental settings, the number of gfp-positive cells decreased proportionately to the amount of virus used. Furthermore, the number of gfp-positive cells increase with time after infection. This is in contrast with the observation by Alexander et $a l^{17}$ for crude lysates of AP-expressing rAAV vectors for which the percentage of AP-positive cells was more than $90 \%$ after 2 days and dramatically decreased with time. Thus, in contrast to $\beta$ galactosidase and alkaline phosphatase, gfp protein produced after transfection of producer cells was not transferred to the target HeLa cells, in our experimental settings. This could be due to (1) the limited half-life of the gfp protein ( $4 \mathrm{~h}$; Tavare, personal communication); and/or (2) the absence of uptake of contaminant transgene products by HeLa cells.

The high transduction efficiency observed with sonicated crude lysates of rAAV-EGFP could thus not be explained by the uptake of gfp contaminants. Therefore, we hypothesized that some component of the sonicated cellular extracts stimulated rAAV-mediated gene transfer and/or expression. Cellular and/or adenoviral factor(s) present in the extracts could enhance the transduction efficiency of recombinant viral particles. Indeed, the transduction efficiency of purified rAAV-EGFP was 
stimulated in trans by sonicated HEK-293 cellular extracts which did not contain the EGFP gene or gene products.

However, using crude viral lysates, about 30\% HeLa cells could be transduced at a multiplicity of 5 IU per cell whereas in the trans-stimulation experiments, only $10 \%$ HeLa cells were $\mathrm{gfp}^{+}$at the same multiplicity. A possible explanation of the higher transduction efficiency of the viral lysate relative to the purified virus plus cellular extracts could be that the association between viral particles and cellular contaminants is more tight in the case of crude viral lysate.

Cellular factor(s) present in the extracts could enhance the capacity of the HeLa cells to bind and/or internalize viral particles. However, HeLa cells have been shown to bind efficiently and internalize AAV2. ${ }^{15,34}$ Whether migration to the nucleus and/or uncoating is limiting has not been shown.

Genistein, ${ }^{14}$ adenovirus E4 ORF6 ${ }^{9,10}$ and UV light ${ }^{10}$ enhance transduction of HeLa cells and stimulate secondstrand synthesis. This suggests that the stimulation of transduction by sonicated cellular extracts could be mediated by factor(s) which are involved in conversion of input viral DNA to double-stranded transcriptionally active DNA. This factor(s) could be a HeLa cellular factor induced or modified by the incubation with the extracts similarly to what occurs after genistein or UV treatment, or it could be a 293 factor which is transferred to the target HeLa cells. In the latter case, it could be a small factor which can penetrate the cells without transfection agents or a larger factor (protein, nucleic acid) which penetrated HeLa cells by endocytosis, eg by means of lipid vesicles formed by sonication.

Treatments which stimulate transduction by rAAV vectors have in common to interfere with the metabolism of nucleic acids, possibly through induction of a general cellular response to stress similar to the SOS system in bacteria. ${ }^{35}$ Several proteins are known to be induced by various kinds of stress including viral infection, radiation, carcinogens and cell synchronizing agents. These proteins, such as HSP70 or p53, have pleiotropic effects which can lead to resistance to stress or, when the damage is too important, induce apoptosis to safe-guard the entire organism rather than maintaining a mutated cell. This possible relation between rAAV-mediated transduction and treatments interfering with DNA synthesis is consistent with the hypothesis that second-strand synthesis is the limiting step in transduction, ${ }^{9,10}$ of HeLa cells. Recently, 293 cells were shown to support limited helper-independent AAV replication. ${ }^{36}$ These data could indicate that 293 cells contain factor(s) required for second-strand synthesis, which would be present in limiting concentrations in HeLa cells.

\section{Materials and methods}

\section{Plasmids, cell lines and viruses}

The AAV vector plasmid, pTR-EGFP (Figure 1) containing the enhanced humanized gfp (EGFP) gene has been constructed from pTR-UF, a plasmid vector, kindly given by $S$ Zolotukhin, containing the gene coding for the jellyfish green fluorescent protein (gfp10) under the control of the CMV promoter, as well as the neomycin resistance (neo) gene under the control of the HSVTK promoter. ${ }^{37}$ Briefly, we constructed a derivative of phGFP-S65T
(Clontech, Palo Alto, CA, USA) by replacing the HindIIINotI fragment containing the hGFP-S65T coding sequence (humanized gfp) with the HindIII-NotI fragment from pEGFP-1 (Clontech) containing the EGFP gene, a variant of the humanized gfp with enhanced fluorescence. The gfp operon from pTR-UF (fragment BglIIXhoI) was then replaced by the EGFP operon from this new construct (MluI-BamHI fragment). pIM45, a plasmid containing the AAV coding regions (map position 190 to $4489)^{38}$ was a kind gift from Dr N Muzyczka (University of Florida).

HEK-293, HeLa and CV1 cell lines were purchased from the ATCC (Bethesda, MD, USA). The SV40 T antigen-transformed 293/T cell line originated from Q-one Biotech (Glasgow, UK). The 911 cell line ${ }^{39}$ was a kind gift from Dr Bout (Introgene, Leiden, The Netherlands). All cell lines were cultured in Dulbecco's modified Eagle's medium (DMEM; Gibco BRL, Life Technologies, Merelbeke, Belgium) supplemented with $10 \%$ fetal calf serum, penicillin, streptomycin and non-essential amino acids.

Adenovirus type 2 was a kind gift from Professor E Winocour (Weizmann Institute of Science, Israel), and stocks were prepared as described by Yakobson et al. ${ }^{3}$ Wild-type AAV was prepared by transfection of the pSM620 plasmid containing the entire AAV genome ${ }^{40}$ into adenovirus-preinfected HeLa cells as described by Yakobson et al. ${ }^{3}$

Recombinant adenovirus containing the enhanced humanized gfp (EGFP) gene under the control of the CMV promoter (rAd-EGFP) was a kind gift from Dr B Pichon (IRIBHN), Université Libre de Bruxelles, Belgium). ${ }^{41}$

\section{Recombinant AAV (rAAV) viral preparations}

The recombinant $\mathrm{rAAV}$ EGFP virus was produced by transfection of HEK-293 (10 $10^{6}$ cells per $10 \mathrm{~cm}$ plate seeded 1 day before) with $3 \mu \mathrm{g}$ of pIM45 and $1 \mu \mathrm{g}$ of pTR-EGFP, using the calcium-phosphate coprecipitation method. Twenty-four hours after transfection, the cells were infected ( $2 \mathrm{~h}$ in serum-free medium) with $2 \mathrm{ml}$ adenovirus type 2 at a multiplicity of 3 plaque-forming units (p.f.u.) per cell and the cultures were refed with $8 \mathrm{ml}$ of complete medium. The transfection efficiency of the pTREGFP plasmid, as evaluated by FACS analysis 2 days after transfection, varied between 10 and 40\%. Forty-eight to $60 \mathrm{~h}$ after infection, the cells were further processed according to one of the following protocols.

\section{Crude lysates}

The cells were harvested and centrifuged and the cell pellet was resuspended in $1 \mathrm{ml}$ phosphate buffer saline (PBS) per plate. The cells were disrupted by sonication according to Rolling and Samulski. ${ }^{42}$ Adenovirus was then inactivated by heating at $56^{\circ} \mathrm{C}$ for $30 \mathrm{~min}$. Cellular debris were eliminated by centrifugation at $1500 \mathrm{~g}$ for 5 min.

\section{Purified preparations}

The cells were harvested, centrifuged and the cell pellets from five plates were washed with PBS $\mathrm{pH}$ 6.9, resuspended in $10 \mathrm{ml}$ Tris $50 \mathrm{~mm}$, EDTA $1 \mathrm{~mm}\left(\mathrm{~T}_{50} \mathrm{E}_{1}\right), \mathrm{pH} 8.0$ and submitted to five cycles of freeze-thawing. Sonication was not used in this protocol because it resulted in the release of large amounts of cellular contaminants which interfered with the establishment of the gradient 
(data not shown). Cesium-chloride in $\mathrm{T}_{50} \mathrm{E}_{1} \mathrm{pH} 8.0$ was added up to a final density of $1.41 \mathrm{~g} / \mathrm{cm}^{3}$ and the lysate was further homogenized using a Pasteur pipet and finally forced through a $23 \mathrm{G}$ needle.

Recombinant rAAV-EGFP viral particles obtained from 30 plates were concentrated and purified by two rounds of $\mathrm{CsCl}$ gradients in a Beckmann SW41 rotos (Beckmann, Fullerton, CA, USA) at $154000 \mathrm{~g}$ for $48 \mathrm{~h}$. Positive fractions were dialyzed in microcollodion tubes (Sartorius, Goettingen, Germany) and further concentrated in 50$300 \mu \mathrm{l}$ PBS on Amicon Centricon 30 membranes (Millipore, Bedford, MA, USA) as described by Avalosse et $a l^{43}$ for the purification of MVMp-based parvoviral vectors. Adenovirus was inactivated by heating at $56^{\circ} \mathrm{C}$ for $30 \mathrm{~min}$.

\section{Cellular extracts}

Cells $\left(10^{6}\right.$ cells seeded per $10 \mathrm{~cm}$ plate) were harvested 4 days after seeding and after centrifugation, the cell pellet was resuspended in $1 \mathrm{ml}$ PBS. The cells were disrupted by sonication and heated at $56^{\circ} \mathrm{C}$ for $30 \mathrm{~min}$. The cellular debris were eliminated by centrifugation at $1500 \mathrm{~g}$ for 5 min.

When specified, cells were transfected with $3 \mu \mathrm{g}$ of pIM45 1 day after seeding and/or infected with adenovirus 2 days after seeding.

For the preparation of the 'pseudostocks', cells were transfected with $1 \mu \mathrm{g}$ of pTR-EGFP 1 day after seeding and infected with adenovirus 2 days after seeding. The transfection efficiency, measured as the percent gfp $^{+}$cells 2 days after transfection, was similar in the absence (pseudostock) and in the presence (viral stock) of pIM45.

\section{Infectious titer assay}

The infectious titer assay is a biological test revealing only replication-competent genomes in the rAAV preparation. Serial dilutions of the rAAV preparations were used to infect HeLa cells in the presence of wild-type AAV and adenovirus, both in excesses to provide AAV regulatory and structural genes as well as adenoviral helper functions. ${ }^{3}$ Briefly, $60 \mathrm{~mm}$ diameter dishes were seeded with $1.2 \times 10^{6} \mathrm{HeLa}$ cells. Twenty-four hours later, the cells were infected with $0.5 \mathrm{ml}$ of DMEM medium without serum, containing $10^{8}$ p.f.u. of CsCl-purified adenovirus, $2.0 \times 10^{8}$ infectious units (IU) of CsCl-purified wild-type AAV, and serial dilution of rAAV-EGFP. After $2 \mathrm{~h}$ at $37^{\circ} \mathrm{C}$ for virus adsorption, $4 \mathrm{ml}$ of serum-supplemented medium were added. Twenty-eight hours after infection, cells were transferred to nitrocellulose filters, alkali denatured and hybridized with a ${ }^{32} \mathrm{P}$-labeled neo DNA probe. Foci of cells synthesizing rAAV-EGFP DNA were visualized after autoradiography for $24 \mathrm{~h}$ at $-70^{\circ} \mathrm{C}$. Titers were expressed as infectious units per milliliter $(\mathrm{IU} / \mathrm{ml})$.

\section{In vitro transduction assays}

Microscopic detection of gfp $^{+}$cells: Twenty-thousand HeLa cells per well were seeded on eight-well chamber slides (Lab-TEK, Nalge Nunc, Naperville, IL, USA), 1 day before infection. Cells were infected with dilutions of the rAAV-EGFP preparations in $100 \mu \mathrm{l}$ of DMEM medium without serum. The transduction efficiency was evaluated 2 days after infection, except when otherwise specified. The percentage of gfp-positive cells was determined as the ratio of life fluorescent cells (photographed using a Zeiss Axiophot-2 microscope (Zeiss, Jena, Germany) equipped with a UV lamp and an FITC filter) and total number of cells (photographed under visible light microscopy) in given representative areas.

Detection of gfp by fluorescence-activated cell sorter (FACS): Fifty-thousand HeLa cells per well were seeded in 24-well plates, 1 day before infection. Cells were infected with dilutions of the rAAV-EGFP preparations in $100 \mu \mathrm{l}$ of DMEM medium without serum. The transduction titer was evaluated 2 days after infection, except when otherwise specified. Cells were trypsinized and $10^{4}$ cells were analyzed using a FACS scan and the Lysis II software (Becton Dickinson, Mountain View, CA, USA).

\section{Stereotaxy}

Adult male Sprague-Dawley rats (Iffa Credo, L'Arbresle, France) were used for unilateral intracerebral injections as previously described. ${ }^{44}$ Briefly, $2 \mu$ l of purified rAAVEGFP were infused using a motor-driven syringe $(0.2 \mu \mathrm{l}$ per min) connected to a $31 \mathrm{G}$ needle. Animals were killed 1 month after surgery and perfused with $4 \%$ paraformaldehyde in $0.1 \mathrm{~m}$ phosphate buffer. After overnight postfixation in $4 \%$ paraformaldehyde, the brains were rinsed with phosphate buffer and kept at $4^{\circ} \mathrm{C}$.

\section{Immunohistochemistry}

Vibratome sections $(50 \mu \mathrm{m})$ were sequentially incubated in: (1) $3 \% \mathrm{H}_{2} \mathrm{O}_{2}, 10 \%$ methanol in TBS (Tris $10 \mathrm{~mm}, 0.9 \%$ $\mathrm{NaCl}, \mathrm{pH}$ 7.6) for $30 \mathrm{~min}$; (2) THST (50 mm Tris, $0.5 \mathrm{~m}$ $\mathrm{NaCl}, 0.5 \%$ triton $\mathrm{X} 100 \mathrm{pH} 7.6$ ) for $30 \mathrm{~min}$; (3) polyclonal rabbit anti-gfp (Clontech) diluted 1:250 in THST overnight at $4^{\circ} \mathrm{C}$; (4) donkey anti-rabbit-biotin (Amersham, Belgium) 1:200 in TBS, $1 \mathrm{~h}$ at room temperature; (5) streptavidin coupled to horse radish peroxidase, 1:200 in TBS. The peroxidase staining was revealed using the $\mathrm{ABC}$ Elite vectastain kit and diaminobenzidine (NTL Laboratories, Brussels, Belgium), according to the manufacturer's protocol. Sections were mounted in gelatincoated slides, dehydrated and mounted using Entellan mounting fluid (Merck, Darmstadt, Germany). Sections were photographed using a Zeiss Axiophot 2 microscope.

\section{Acknowledgements}

We thank J Brotchi for constant support. We are grateful to $\mathrm{M}$ Pechanski for helpful discussions and to E Winocour for critical reading of this manuscript. We are also grateful to W Hermens and J Verhaagen for their help in the histological analysis of brain sections and to B Pichon for the gift of rAd-EGFP viral stock. We thank $C$ Melas for excellent technical assistance, $C$ Bruyns for her help in the FACS analysis, and J-C Dumon for photographic work. This work was supported by EC grant No. BioCT97-2207 and a grant from the Belgian National Research Foundation (FRSM No. 3.4565.98). MH was a recipient of a postdoctoral fellowship from Université Libre de Bruxelles. MP was a recipient of a Télévie fellowship from the Belgian National Research Foundation (FNRS). 


\section{References}

1 Weitzmann M, Fisher KJ, Wilson JM. Recruitment of wild-type and recombinant adeno-associated virus into adenovirus replication centers. J Virol 1996; 70: 1845-1854.

2 Yakobson B, Koch T, Winocour E. Replication of adeno-associated virus in synchronized cells without the addition of a helper virus. J Virol 1988; 61: 972-981.

3 Yakobson B, Hrynko B, Peak MJ, Winocour E. Replication of adeno-associated virus in cells irradiated with UV light at 254 nm. J Virol 1989; 63: 1023-1030.

4 Ward P, Dean FB, O'Donnel ME, Berns K. Role of the adenovirus DNA-binding protein in in vitro adeno-associated virus DNA replication. J Virol 1998; 72: 420-427.

5 Beaton A, Palumbo P, Berns K. Expression from the adeno-associated virus p5 and p19 promoters is negatively regulated in trans by the rep protein. J Virol 1989; 63: 4450-4454.

6 Zhou X, Muzyczka N In vitro packaging of adeno-associated virus DNA. J Virol 1998; 72: 3241-3247.

7 Schlehöfer JR, Ehrlar M, Zur Hausen H. Vaccinia virus, herpes simplex virus and carcinogens induce DNA amplification in a human cell line and support replication of a helper virus dependent parvovirus. Virology 1986; 152: 110-117.

8 Yalkinoglu AO et al. DNA amplification of adeno-associated virus as a response to genotoxic stress. Cancer Res 1986; 48: 3123-3129.

9 Fisher KJ et al. Transduction with recombinant adeno-associated virus for gene therapy is limited by leading-strand synthesis. J Virol 1996; 70: 520-532.

10 Ferrari FK, Samulski T, Weitzmann M, Samulski RJ. Secondstrand synthesis is a rate-limiting step for efficient transduction by recombinant adeno-associated virus vectors. J Virol 1996; 70: 3227-3234.

11 Russell DW, Miller AD, Alexander IE. Adeno-associated virus vectors preferentially transduce cells in the $S$ phase. Proc Natl Acad Sci USA 1994; 91: 8915-8919.

12 Alexander IE, Russell DW, Spence AM, Miller AD. Effects of gamma irradiation on the transduction of dividing and nondividing cells in brain and muscle of rats by adeno-associated virus vectors. Hum Gene Ther 1996; 7: 841-850.

13 Russell DW, Alexander IE, Miller AD. DNA synthesis and topoisomerase inhibitors increase transduction by adeno-associated virus vectors. Proc Natl Acad Sci USA 1995; 92: 5719-5723.

14 Qing K et al. Role of tyrosine phosphorylation of a cellular protein in adeno-associated virus 2-mediated transgene expression. Proc Natl Acad Sci USA 1997; 94: 10879-10884.

15 Qing $\mathrm{K}$ et al. Adeno-associated virus type 2-mediated gene transfer: correlation of tyrosine phosphorylation of the cellular single-stranded D sequence binding protein with transgene expression in human cells in vitro and in murine tissues in vivo. I Virol 1998; 72: 1593-1599.

16 Ponnazhagan S et al. Adeno-associated virus 2-mediated gene transfer in vivo: organ-tropism and expression of transduced sequences in mice. Gene 1997; 190: 203-210.

17 Alexander IE, Russell DW, Miller AD. Transfer of contaminants in adeno-associated virus vector stocks can mimic transduction and lead to artefactual results. Hum Gene Ther 1997; 8: 1911-1920.

18 Kapplitt MG et al. Long-term gene expression and phenotype correction using adeno-associated virus vectors in the mammalian brain. Nat Genet 1994; 8: 148-154.

19 Mandel RJ, Spratt SK, Snyder RO, Leff SE. Midbrain injection of recombinant adeno-associated virus encoding rat glial cell linederived neurotrophic factor protects nigral neurons in a progressive 6-hydroxydopamine-induced degeneration model of Parkinson's disease in rats. Proc Natl Acad Sci USA 1997; 94: 14083-14088.

20 McCown T et al. Differential and persistent expression patterns of CNS gene transfer by adeno-associated virus (AAV) vector. Brain Res 1996; 713: 99-107.

21 Peel AL, Schrimsher GW, Muzyczka N, Reier PJ. Efficient transduction of green fluorescent protein in spinal cord neurons using adeno-associated virus vectors containing cell typespecific promoters. Gene Therapy 1997; 4: 16-24.

22 Xiao $\mathrm{X}$ et al. Adeno-associated virus (AAV) vector antisense gene transfer in vivo decrease GABAA $\alpha 1$ containing receptors and increases inferior collicular seizure sensitivity. Brain Res 1997; 756: 76-83

23 Klein RL et al. Neuron-specific transduction in the rat septohippocampal or nigrostriatal pathway by recombinant adenoassociated virus vectors. Exp Neurol 1998; 150: 183-194.

24 Bartlett JS, Samulski RJ, McCown TJ. Selective and rapid uptake of adeno-associated virus type 2 in brain. Hum Gene Ther 1998; 9: 1181-1186.

25 Podsakoff G, Wong KK, Chaterjee S. Efficient gene transfer into nondividing cells by adeno-associated virus-based vectors. J Virol 1994; 68: 5656-5666.

26 Samulski RJ, Chang LS, Shenk T. Helper-free stocks of recombinant adeno-associated viruses: normal integration does not require viral gene expression. J Virol 1989; 63: 3822-3828.

27 Snyder R, Xiao X, Samulski RJ. Production of recombinant adeno-associated viral vectors. In: Dracopoli $\mathrm{N}$ et al (eds). Current Protocols in Human Genetics. John Wiley: New York, 1996, pp 12.1.1-12.1.24.

28 Tamayose K, Hirai Y, Shimada T. A new strategy for large scale preparation of high titer recombinant adeno-associated virus vectors by using packaging cell lines and sulfonated cellulose column chromatography. Hum Gene Ther 1996; 7: 507-513.

29 Chiorini JA et al. High-efficiency transfer of the T cell co-stimulatory molecule B7-2 to lymphoid cells using high-titer recombinant adeno-associated virus vectors. Hum Gene Ther 1995; 6: 1531-1541.

30 Clark KR, Voulgaropoulou F, Johnson PR. Cell lines for the production of recombinant adeno-associated virus. Hum Gene Ther 1996; 6: 1329-1341.

31 Xiao X, Samulski RJ. Production of high-titer recombinant adeno-associated virus vectors in the absence of helper adenovirus. J Virol 1998; 72: 2224-2232.

32 Clark KR, Johnson PR. A stable cell line carrying adenovirusinducible rep and cap genes allows for infectivity titration of adeno-associated virus vectors. Gene Therapy 1996; 3: 1124-1132.

33 Salvetti A et al. Factors influencing recombinant adeno-associated virus production. Hum Gene Ther 1998; 9: 695-706.

34 Summerford C, Samulski RJ. Membrane-associated heparan sulfate proteoglycan is a receptor for adeno-associated virus type 2 virions. J Virol 1998; 72: 1438-1445.

35 Radman M. Is there SOS induction in mammalian cells? Photochem Photobiol 1980; 32: 823-830.

36 Wang X-S, Srivastava A. Rescue and autonomous replication of adeno-associated virus type 2 genomes containing Rep-binding site mutations in the viral p5 promoter. J Virol 1998; 72: 4811-4818.

37 Zolotukhin $\mathrm{S}$ et al. A humanized green fluorescent protein cDNA adapted for high-level expression in mammalian cells. I Virol 1996; 70: 4646-4654

38 McCarty DM, Christensen M, Muzyczka N. Sequences required for the coordinate induction of the AAV p19 and p40 promoters by the Rep protein. J Virol 1991; 68: 4988-4997.

39 Fallaux F et al. Characterization of 911: a new helper cell line for the titration and propagation of early region 1-deleted adenoviral vectors. Hum Gene Ther 1995; 7: 215-222.

40 Samulski RJ, Berns K, Tan KI, Muzyczka N. Cloning of adenoassociated virus into pBR322: rescue of intact virus from the recombinant plasmid in human cells. Proc Natl Acad Sci USA 1982; 79: 2077-2081.

41 Pichon B. PhD thesis. Université Libre de Bruxelles, 1998

42 Rolling F, Samulski RJ. AAV as a viral vector for human gene therapy. Molec Biotech 1995; 3: 9-15.

43 Avalosse B et al. Method for concentrating and purifying recombinant autonomous parvovirus vectors designed for tumourcell-targeted gene therapy. J Virol Meth 1996; 62: 179-183.

44 Levivier M, Przedborski S, Bencsics C, Kang UJ. Intrastriatal implantation of fibroblasts genetically engineered to produce brain-derived neurotrophic factor prevents degeneration of dopaminergic neurons in a rat model of Parkinson's disease. J Neurosci 1995; 15: 7810-7820. 